\title{
VOXES: A NEW HAPG MOSAIC CRYSTAL BASED VON HAMOS SPECTROMETER FOR MILLIMETRIC SOURCES
}

\author{
A. Scordo ${ }^{1 *}$, V. De Leo ${ }^{2}$, C. Curceanu', M. Miliucci1 ${ }^{*}$, F. Sirghi' \\ ${ }^{1}$ Laboratori Nazionali di Frascati INFN, Frascati (Rome), Italy \\ ${ }^{2}$ Università degli Studi di Roma "La Sapienza”, Rome, Italy
}

\begin{abstract}
Von Hamos (VH) spectrometers are widely used in several fields, ranging from fundamental physics to very different types of practical applications. However, these type of Bragg spectrometers are usually operated in high rate - high resolution experiments, where the typical source size can be as low as few tens of microns. The VOXES collaboration at the INFN Laboratories of Frascati recently developed a VH spectrometer, making use of Highly Annealed Pyrolitic Graphite (HAPG) mosaic crystals and an X-ray beam optics optimization, which could be used for source sizes up to few $\mathrm{mm}$ in the Bragg plane, some tens of $\mathrm{mm}$ in the sagittal plane and, if gaseous sources are used, several tens of $\mathrm{cm}$ in the X-ray propagation direction. Such kind of spectrometer could be used in a wide range of applications in several fields, going from fundamental physics, synchrotron radiation and $X$-FEL applications, astronomy, medicine and industry to hadronic physics experiments, measuring exotic atoms with extremely high precision. We present the results obtained with the VOXES spectrometer, in terms of spectral resolution and achievable precision, for a $206.7 \mathrm{~mm}$ curvature radius HAPG crystal using Fe Ko1,2 transition lines.
\end{abstract}

Keywords: $H A P G$, mosaic crystals, Von Hamos spectrometer, effective source size, Bragg spectroscopy, X-ray spectroscopy

\section{INTRODUCTION}

High precision measurements of X-rays are important in many fields of fundamental science, from particle and nuclear physics to quantum mechanics, as well as in astronomy and in several applications using synchrotron light sources or X-FEL beams, in biology, medicine and industry. An intensive effort has been pursued in the last years to optimize and develop large area X-ray detection systems; new types of detectors are being constantly realized, with improved performances in terms of efficiency, energy resolution and costs.

Still, when few eV linewidths must be measured or precisions below $1 \mathrm{eV}$ are needed, standard solid-state devices are limited by their intrinsic resolution of about $120 \mathrm{eV}$ (FWHM) at $6 \mathrm{keV}$, related to the Fano Factor and to the electronic noise.

An alternative is represented by Bragg spectroscopy, one of the best-established highresolution X-ray measurement techniques, where the X-ray energy spectrum can be obtained exploiting photon reflection on crystals following the Bragg rule $\mathrm{n} \lambda=2 \mathrm{~d} \sin \theta$, where $\mathrm{n}$ is an integer number (order of reflection), $\lambda$ is the photon wavelength, $d$ is the crystal lattice constant and $\theta$ is the photon impinging angle on the crystal surface.

If a monochromatic parallel X-ray beam must be measured, the spectrum is obtained by several acquisitions with different $\theta$ values around the nominal Bragg angle $\theta_{\mathrm{B}}$; on the contrary, if one needs to measure a polychromatic or isotropic photon source, the spectrum is obtained in one shot with a single $\theta$ using a position detector.

In the first case, higher resolutions can be obtained with the drawback of a longer data taking; in the second case, in which the measurements presented in this work fall into, the faster exposure time is balanced by a higher background due to the beam divergence and the source size. Therefore, when the photons emitted from extended isotropic sources (like a gaseous or liquid target) must be measured, this method has been until now ruled out by the constraint to reduce the dimension of the target to a few tens of microns $[1,2]$.

In the last decades, the development of Pyrolitic Graphite (PG) mosaic crystals [3-5] renewed the interest in Bragg spectrometers as possible candidates to measure millimetric and isotropic X-ray sources. Mosaic crystals consist of many nearly perfect small PG crystallites, randomly misoriented around the lattice main direction; the FWHM of this angular distribution, called mosaicity $(w)$, makes it possible that even a photon not reaching the crystal with the exact Bragg energy-angle relation can find a properly oriented crystallite and be reflected [6]. This, together with a lattice spacing constant of $3.514 \AA$, enables them to be highly efficient in diffraction in the $2-20 \mathrm{keV}$ energy range, for the $n=1$ reflection order, while higher energies can be reached at higher orders.

*alessandro.scordo@lnf.infn.it, marco.miliucci@lnf.infn.it 
From the definition of mosaicity, it follows that those photons which find a properly oriented crystallite in the inner part of the crystal are reflected on the position detector slightly shifted with respect to the one coming from a surface crystallite; because of this defocusing process, the peak resolution is worsened with respect to a standard non-mosaic crystal. For the same principle, for a given mosaicity, the thickness of the graphite crystal plays a fundamental role; for higher thickness, the reflection efficiency is enhanced at the expense of the peak resolution [7-11].

Thanks to two different production mechanisms, Pyrolitic Graphite crystals can be obtained with different mosaicity and divided into two main families: Highly Annealed Pyrolitic Graphite (HAPG) and Highly Oriented Pyrolitic Graphite (HOPG) crystals, showing lower and higher mosaicities, with consequently higher and lower resolutions, respectively. Both crystal types can be realized with different ad hoc geometries, making them suitable to be used in the VH configuration [12], combining the dispersion of a flat crystal with the focusing properties of cylindrically bent ones.

Von Hamos spectrometers have been extensively used in the past providing very promising results in terms of spectral resolution but all the available works in literature report measurements for effective source dimensions of some tens of microns or parallel and monochromatic beams; these configurations are achieved either with micro focused X-ray tubes, or with a set of slits and collimators placed before the target to minimize the activated area. Spectrometer versions based on a full-cylinder geometry, to be used with a pixelated area detector instead of a one-dimensional strip detector, have been also developed. This may lead to an increase in both spectral resolution, thanks to a better integration of the bent lines produced by the crystal, and overall efficiency, thanks to a higher solid angle acceptance, but the effect of a hundred of microns source size are still to be tested [14].

The aim of the VOXES project at INFN Laboratories of Frascati (LNF) is to realize a HAPG based VH spectrometer able to achieve challenging resolutions of few $\mathrm{eV}$ even with sources of dimensions, in the Bragg plane, of some millimeters.

In this work we present, as an example of the results obtained with the VOXES spectrometer, the energy resolutions and the peak positions precisions obtained measuring $\mathrm{Fe}$ Ka1,2 transitions, using a 206.7 mm radius of curvature HAPG crystal, for different effective source sizes and angular acceptances.

\section{EXPERIMENTAL APPARATUS AND X-RAY BEAM PARAMETERS}

\subsection{Description of the apparatus}

In Figure 1, a drawing of the experimental apparatus used for our measurements is shown.

A thin foil (target) is placed inside an aluminum box and activated by a XTF-5011 X-ray tube, produced by Oxford Instruments, placed on top of the box; the center of the foil, placed on a $45^{\circ}$ rotated support prism, represents the origin of the reference frame in which $\mathrm{Z}$ is the direction of the characteristic photons emitted by the target and forming, with the $\mathrm{x}$-axis, the Bragg reflection plane, while $y$ is the vertical direction, along which primary photons generated by the tube are shot. Two adjustable motorized slits (STANDA 10AOS10-1) with $1 \mu \mathrm{m}$ resolution are placed after the $5.9 \mathrm{~mm}$ diameter circular exit window of the aluminum box to shape the outcoming X-ray beam. The used HAPG crystals, produced by the OPTIGRAPH company in Berlin, Germany [4], are deposited on different curvature radii Thorlabs N-BK7 $30 \times 32 \mathrm{~mm}^{2}$ uncoated Plano-Concave Cylindrical lenses, held by a motorized mirror mount (STANDA 8MUP21-2) with a double axis $<1$ arcsec resolution and coupled to a 0.01 $\mu \mathrm{m}$ motorized vertical translation stage (STANDA 8MVT40-13), a 4.5 arcsec resolution rotation stage (STANDA 8MR191-28) and two motorized $0.156 \mu \mathrm{m}$ linear translation stages (STANDA 8MT167-25LS). The position detector is a commercial MYTHEN2-1D 640 channels strip detector produced by DECTRIS (Zurich, Switzerland), having an active area of $32 \times 8 \mathrm{~mm}^{2}$, and whose strip width and thickness are, respectively, $50 \mu \mathrm{m}$ and $420 \mu \mathrm{m}$. The MYTHEN2-1D detector is also coupled to a positioning motorized system identical to the one of the HAPG holder. Finally, a standard Peltier Cell is kept on top of the strip detector to stabilize its temperature in the working range of $18^{\circ}-28^{\circ}$. The resulting 10 -axis motorized positioning system is mounted on a set of DRYLIN rails and carriers to ensure better stability and alignment and, in addition, to easily adjust source-crystal-detector positions for each energy to be measured.

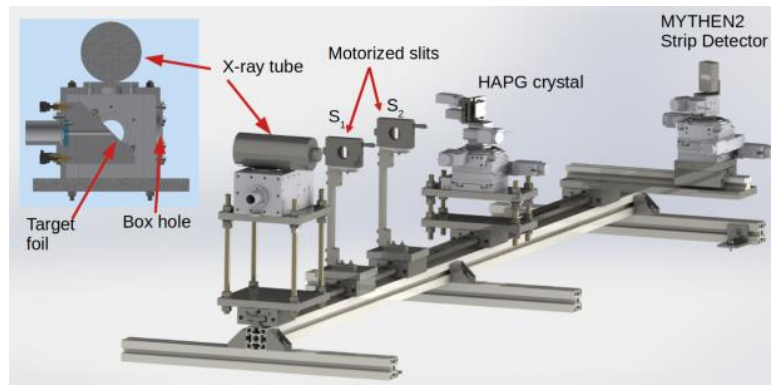

Figure 1. Drawing of the experimental apparatus.

\subsection{X-ray beam parameters: effective source size} and angular acceptance

As pointed out in the Introduction, the actual limitation on the possible usage of crystal spectrometers for extended targets is represented by the requirement of a point-like source; however, using a pair of slits as the one described in the previous section, it is possible to shape the beam of X-rays emitted by an extended and diffused target in such a way as to simulate a virtual point-like source.

In Figure 2, a schematic of the beam parameters in the Bragg dispersion plane is presented.

Referring to Figure 2, this configuration is obtained setting the position (z1 and z2) and the aperture ( $\mathrm{S} 1$ and $\mathrm{S} 2$ ) of each slit to create a virtual source between them ( $\mathrm{z}$, green solid lines on Figure 2), an angular acceptance $\Delta \theta$, and an effective source $S_{o}$ (green) which can be, in principle, as wide as 
necessary. The $\Delta \theta$ angular acceptance could also be set to any value, provided it is large enough to ensure that all the $\theta_{\mathrm{B}}$ corresponding to the lines to be measured are included. For example, to measure in a single spectrum $\mathrm{Fe} \operatorname{K} \alpha 1\left(\theta_{\mathrm{B}}=16.774^{\circ}\right)$ and $\mathrm{Fe} \operatorname{K} \alpha 2\left(\theta_{\mathrm{B}}=16.809^{\circ}\right)$, a $\Delta \theta \geq 0.035^{\circ}$ is required.

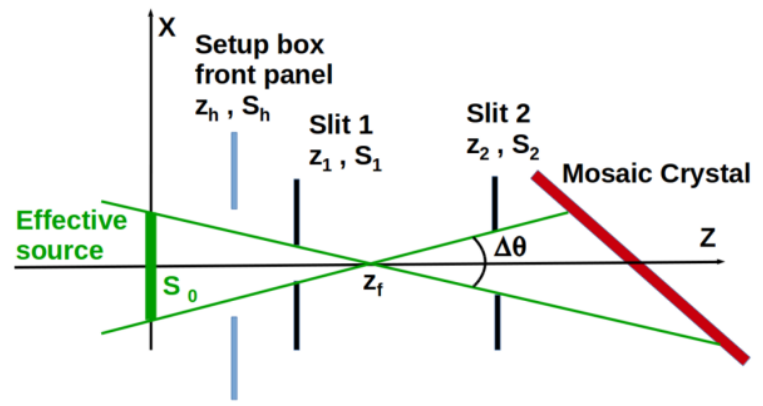

Figure 2. X-ray beam geometry on Bragg dispersion plane

In this work we show a scan of both the angular acceptance and the effective source size; the main scope is to check how big the latter can be set without degrading the resolution too much.

For further details about the X-ray beam geometry, as well as for a full description also of the focusing properties of the VOXES spectrometer in the vertical (sagittal) plane, we refer to our previous work [15].

\section{DATA ANALYSIS AND RESULTS}

In this section we present the spectra obtained for $\mathrm{Fe}$ Ka1,2 transitions using a $206.7 \mathrm{~mm}$ curvature radius HAPG crystals. For each energy, since the source-crystal-detector are fixed by the $\mathrm{VH}$ configuration constraints, slits positions z1 and z2 are chosen such as to have a vertical dispersion at the HAPG position smaller than the crystal size (30 $\mathrm{mm}$ ).

The scan is performed varying the slits openings to obtain different values of the $\mathrm{S}_{o}$ and $\Delta \theta$ parameters and keeping a fixed integration time of 60 minutes to compare the resuls also in terms of achievable precision in the peak position (which is strongly dependent on the statistics).

\subsection{Data analysis procedure}

For a given $S_{o}, \Delta \theta$ pair, the Bragg spectrum is collected with the MYTHEN2 strip detector; then, it is

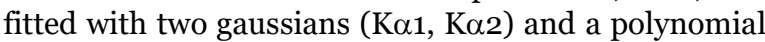
for the background to extract the peak positions and to calibrate it.

We performed, in a previous work, an extensive study of both the best fitting and the best calibration functions to be used [15] and we refer to it for the results shown in this paper.

Finally, for each $S_{o}, \Delta \theta$ pair the peak resolutions $(\sigma)$, the rates and the precisions on the peak positions are extracted from the fits to the calibrated spectra and compared.

\subsection{Results}

In Figures 3-8, we report the Fe Ka1,2 rates, the common gaussian peak resolution $\sigma$ (see [15]), the obtained peak position precisions and an example of a fitted spectrum, respectively. In this example, the individual peak fitting functions $\mathrm{K} \alpha 1$ and $\mathrm{K} \alpha 2$ correspond to the green and violet curves, respectively, and are, together with a polynomial background (blue) and the overall fit (red), superimposed on the calibrated spectrum.

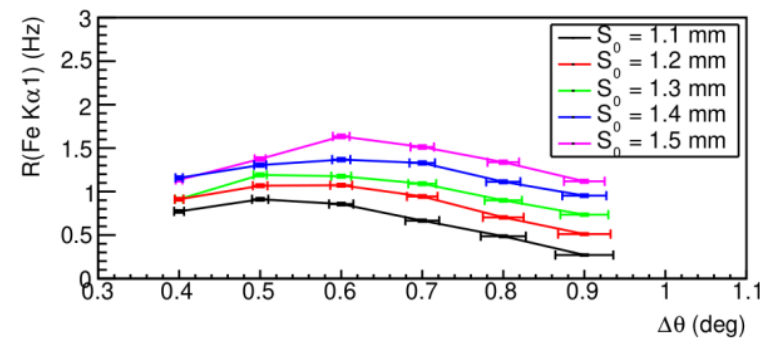

Figure 3. Fe K $\alpha 1$ signal rates; $S_{o}$ and $\Delta \theta$ are the effective source size and angular acceptance described in Sec. 2.2.

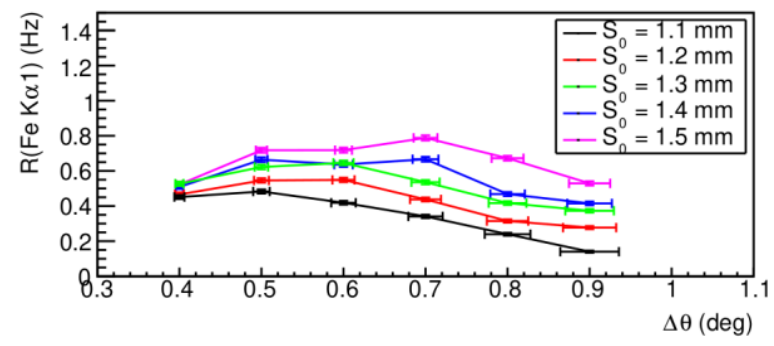

Figure 4. Fe K $\alpha 2$ signal rates; $S_{o}$ and $\Delta \theta$ are the effective source size and angular acceptance described in Sec. 2.2.

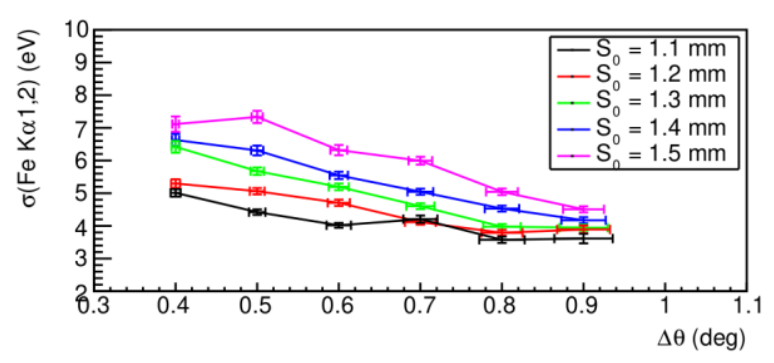

Figure 5. Fe K $\alpha 1,2$ common $\sigma ; \mathrm{S}_{o}$ and $\Delta \theta$ are the effective source size and angular acceptance described in Sec. 2.2.

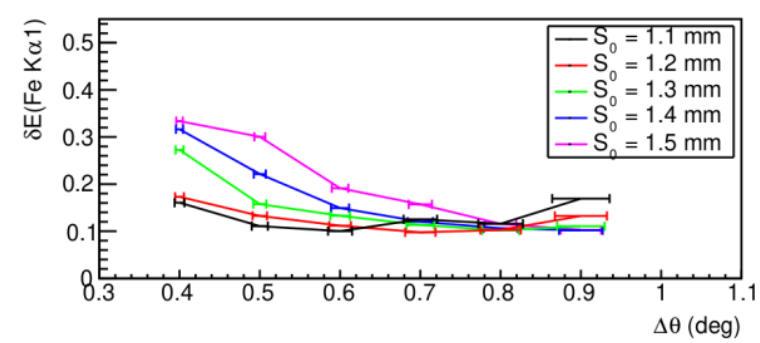

Figure 6. Fe Ko1 peak position precisions; $\mathrm{S}_{\circ}$ and $\Delta \theta$ are the effective source size and angular acceptance described in Sec.2.2. 


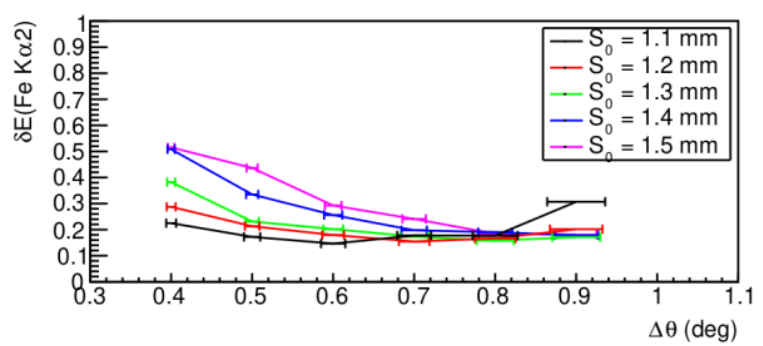

Figure 7. Fe Ka2 peak position precisions; $\mathrm{S}_{\circ}$ and $\Delta \theta$ are the effective source size and angular acceptance described in Sec. 2.2 .

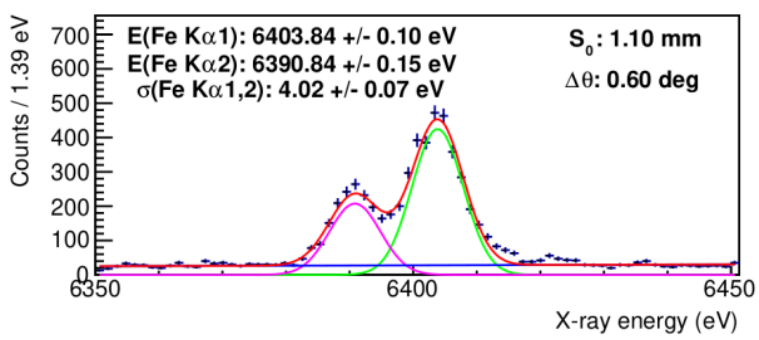

Figure 8. Example of a fitted calibrated spectrum obtained with $\mathrm{S}_{0}=1.1 \mathrm{~mm}$ and $\Delta \theta=0.6^{\circ}$. Ka1, Ka2, polynomial background and total fitting function correspond to the green, violet, blue and red curves, respectively.

These plots show how, for a given crystal and for a given integration time, an optimal $S_{o}, \Delta \theta$ pair can be found which maximizes the peak precision. This is a very important result because it allows to set the beam geometry in the best configuration in any possible application.

The presence of a minimum in the plots, despite of a constantly decreasing $\sigma$, is due to an increase in the number of events ( $\mathrm{N}$ ) for bigger $\Delta \theta$.

It is also very important to underline how, using the two slits geometry, the VOXES spectrometer managed to measure $\mathrm{Fe} \mathrm{K} \alpha 1,2$ transitions with a resolution $(\sigma)$ of the order of $5 \mathrm{eV}$ with an effective source size of $1.5 \mathrm{~mm}$. This is a very encouraging result towards the application of our spectrometer for extended (non-pointlike) sources.

\section{CONCLUSIONS}

In this paper we presented the results obtained with the VOXES Von Hamos spectrometer based on mosaic crystals of pyrolytic graphite (HAPG), with the aim to test its spectroscopic capabilities when used with effective source of many hundreds of microns (up to $1.2 \mathrm{~m}$ ).

We demonstrated how the proposed spectrometer allows to obtain energy resolutions in the order of $0.1 \%$ (FWHM/E), for energies below $10 \mathrm{keV}$; in this work we reported, as an example, measurements of $\mathrm{Fe} \mathrm{K} \alpha 1,2$ transitions with a resolution $(\sigma)$ of the order of $5 \sim 7 \mathrm{eV}$ with an effective source size up to $1.5 \mathrm{~mm}$.

Also, we demonstrated how, for a given energy and crystal radius of curvature, the best X-ray beam configuration can be found to either maximize the energy resolution or the precision on the peak position. 85
These results are very encouraging and promote this spectroscopy technique as a serious candidate for high precision X-ray measurements from diffused and extended sources, with possible applications in several fields, going from fundamental physics, synchrotron radiation and X-FEL applications, astronomy, medicine and industry to hadronic physics experiments, measuring exotic atoms with extremely high precision.

Acknowledgements: This work is supported by the

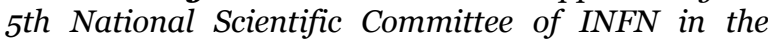
framework of the Young Researcher Grant 2015, no. 17367/2015. This project has received funding from the European Union's Horizon 2020 research and innovation programme under STRONG-2O2O grant agreement No 824093. We thank the LNF and SMI staff, in particular the LNF SPCM service and Doris Pristauz-Telsnigg, for the support in the preparation of the setup.

\section{REFERENCES}

1. H. Legall, et al., "A new generation of X-ray optics based on pyrolitic graphite," in Proc. 28th Int. Conf. Free Electron Laser (FEL 2006), Berlin, Germany, 2006, pp. $798-801$.

Retrieved from:

https://accelconf.web.cern.ch/fo6/PAPERS/FRAAUo4. PDF

Retrieved on: Jan. 12, 2021

2. R. Barnsley et al., "Versatile high resolution crystal spectrometer with X-ray charge coupled device detector," Rev. Sci. Instrum., vol. 74, no. 4, pp. 2388 - 2408, Apr. 2003.

DOI: $10.1063 / 1.1533105$

3. A. A. Antonov et al., "First results on application of short-focus monochromators from formed pyrolytic graphite for X-ray fluorescent analysis using synchrotron radiation," Rev. Sci. Instrum., vol. 60, no. 7, pp. 2462 - 2463, Jul. 1989.

DOI: $10.1063 / 1.1140699$

4. Optigraph: Optigraph References, Berlin, Germany. Retrieved from: http://www.optigraph.eu/references.html\#ref o7 Retrieved on: Jan. 12, 2021

5. M.S. del Rio et al., "Focusing properties of mosaic crystals," in Proc. Int. Symp. Optical Science, Engineering, and Instrumentation (SPIE 1998), San Diego (CA), USA, 1998, pp. 246 - 255. DOI: $10.1117 / 12.332512$

6. M. Gerlach et al., "Characterization of HAPG mosaic crystals using synchrotron radiation," J. Appl. Crystallogr., vol. 48, no. 5, pp. 1381 - 1390, Oct. 2015. DOI: $10.1107 / \mathrm{S} 160057671501287 \mathrm{X}$

7. A. Scordo, C. Curceanu, M. Miliucci, F. Sirghi, J. Zmeskal, "Pyrolitic Graphite Mosaic Crystal Thickness and Mosaicity Optimization for an Extended Source von Hamos X-ray Spectrometer, Condens. Matter, vol. 4, no. 2, 38, Jun. 2019. DOI: $10.3390 /$ condmat4020038

8. G. E. Ice, C. J. Sparks Jr., "Mosaic crystal X-ray spectrometer to resolve inelastic background from anomalous scattering experiments," Nucl. Instrum. Methods Phys. Res. A, vol. 291, no. 1 - 2, pp. 110 - 116, May 1990. DOI: $10.1016 / 0168-9002(90) 90043-6$

9. H. Legall, H. Stiel, V. Arkadiev, A. A. Bjeoumikhov, "High spectral resolution X-ray optics with highly oriented pyrolytic graphite," Opt. Express, vol. 14 no. 10, pp. 4570 - 4576, May 2006. DOI: 10.1364/oe.14.004570 
PMid: 19516609

10. U. Zastrau et al., "Focal aberrations of large-aperture HOPG von-Hamos X-ray spectrometers,” J. Inst., vol. 7, no. 9, Po9015, Sep. 2012.

DOI: $10.1088 / 1748-0221 / 7 / 09 / P 09015$

11. U. Zastrau et al., "Characterization of strongly-bent HAPG crystals for von-Hámos X-ray spectrographs," J. Inst., vol. 8, no. 10, P10006, Oct. 2013. DOI: $10.1088 / 1748-0221 / 8 / 10 /$ P10006

12. L. V. Von Hamos, "Röentgenspektroskopie und Abbildung mittels gekrümmter Kristallreflektoren. I. Geometrisch-optische Betrachtungen," Ann. Phys. (Berl.), vol. 409, no. 6, pp. 716 - 724, Jan. 1933.

(L. V. Von Hamos, "X-ray spectroscopy and imaging using curved crystal reflectors. I. Geometric-optical considerations," Ann. Phys. (Berl.), vol. 409, no. 6, pp. 716 - 724, Jan. 1933.)

DOI: 10.1002/andp.193340906o8
13. A. P. Shevelko, Yu. S. Kasyanov, O. F. Yakushev, L. V. Knight, "Compact focusing von Hamos spectrometer for quantitative X-ray spectroscopy," Rev. Sci. Instrum., vol. 73, no. 10, pp. 3458 - 3463, Oct. 2002. DOI: $10.1063 / 1.1502013$

14. L. Anklamm et al., "A novel von Hamos spectrometer for efficient X-ray emission spectroscopy in the laboratory," Rev. Sci. Instrum., vol. 85, no. 5, 053110, May 2014. DOI: $10.1063 / 1.4875986$

15. A. Scordo et al., "High resolution multielement XRF spectroscopy of extended and diffused sources with a graphite mosaic crystal based Von Hamos spectrometer," J. Anal. At. Spectrom., vol. 35, no. 1, pp. 155 - 168, Jan. 2020.

DOI: 10.1039/C9JA00269C 